\title{
A New Synchronous Reference Frame-Based Method for Single-Phase Shunt Active Power Filters
}

\author{
Mohammad Monfared $^{\dagger}$, Saeed Golestan ${ }^{* *}$, and Josep M. Guerrero ${ }^{* * *}$ \\ †Dept. of Electrical Eng., Faculty of Engineering, Ferdowsi University of Mashhad, Mashhad, Iran \\ *Dept. of Electrical Eng., Abadan Branch, Islamic Azad University, Abadan, Iran \\ *** Dept. of Energy Technology, Aalborg University, Aalborg, Denmark
}

\begin{abstract}
This paper discusses the design of a novel synchronous reference frame (SRF) method that can extract the reference compensating current for single-phase shunt active power filters (APFs). Unlike previous SRF studies, the proposed method has an innovative feature that does not require a fictitious current signal. Other key features of the proposed strategy include frequency-independent operation, accurate reference current extraction, and relatively fast transient response. The effectiveness of the proposed method is investigated by conducting a detailed mathematical analysis. Results of the analysis confirm the superior performance of the suggested approach. Theoretical evaluations are confirmed by the experimental results.
\end{abstract}

Keywords: Active filter, Synchronous reference frame, Power quality

\section{INTRODUCTION}

With the present increasing use of non-linear loads, particularly power electronic equipments, and with emerging power quality issues, both energy consumers and the electrical supply industry demand innovative and cost-effective power quality enhancement solutions. Traditionally, shunt-connected LC passive filters, tuned at dominant harmonic frequencies had been used to absorb harmonic currents generated by non-linear loads. Besides their bulky sizes and heavy weights, these filters suffer from serious technical drawbacks such as (1) susceptibility to resonances with the load and line impedances, (2) sensitivity to component tolerances and system parameter variations, and (3) possibility of overloading by ambient harmonic loads. Active power filters (APFs) have been proven successful in overcoming the above problems. An APF is a power electronic converter-based device designed to compensate current and voltage disturbances caused by non-linear loads. Compared with passive filters, APFs offer superior filtering performance, smaller size, and flexibility in the application. Although the cost of APFs is high, severe harmonic distortion limits in passive filters have drastically

Manuscript received Sep. 7, 2012; revised Apr. 5, 2013

Recommended for publication by Associate Editor Kyo-Beum Lee.

Corresponding Author: m.monfared@um.ac.ir

Tel: +98-511-8805017, Ferdowsi University of Mashhad

** Dept. of Electronic Eng., Abadan Branch, Islamic Azad University, Iran

**** Dept. of Energy Technology, Aalborg University, Denmark increased their installation and operating costs, thus resulting in APFs to be considered as economically viable. Many manufacturers have already placed APFs on the market and cause a strong competition in terms of cost and performance. APFs, consequently, are becoming affordable and can challenge traditional passive filters in high-demand applications [1]. APFs can be classified as either three- or single-phase APFs. The current study focuses on the latter type. Single-phase APFs can be used in low- and medium-power applications, particularly in single-phase adjustable speed motor drives, arc furnaces [2], air-conditioning systems [3], electric tractions [4], electronic fluorescent lamp ballasts [5], and so on. In terms of their function, APFs can also be classified into four major categories, namely, (1) shunt APFs, (2) series APFs, (3) hybrid APFs, and (4) unified power quality conditioners (UPQCs) [6]. Generally, a single-phase shunt APF, which is the subject of the present study, can operate in either perfect harmonic cancellation (PHC) or in unity power factor (UPF) mode [7]. In the PHC mode, the shunt APF compensates the total harmonic contents required by the non-linear load, while, in the UPF mode, the reactive current component drawn by the non-linear load is compensated in addition to harmonic currents. Fig. 1 shows a schematic diagram of a single-phase shunt APF. The control system consists of the following main parts: (1) the reference current generation (RCG) block responsible for the extraction of the total harmonic content and of the reactive component of the distorted current drawn by the 
non-linear load when necessary, (2) the DC-link voltage control block that regulates the active power balance between APF and the grid, and (3) the current control block that generates appropriate gating signals for the voltage source inverter (VSI), allowing the output current of VSI to track the reference command delivered by the RCG block.

Different techniques can be applied to generate the reference-compensating current for single-phase shunt APFs [5]-[13]. Among them, the synchronous reference frame (SRF) or the dq theory, which is widely applied in three-phase systems, is considered as one of the simplest and most preferred techniques. The SRF theory can achieve fast and accurate extraction of the harmonic content and of the reactive component of a distorted current, but the application has only been focused in three-phase APFs. Saitou et al. extended the application of the SRF theory to single-phase APFs [14]. In their method, as shown in Fig. 2, the fundamental component of the single-phase signal is shifted by $90^{\circ}$ to generate a fictitious phase signal. Thus, it is possible to represent the single-phase APF as a pseudo two-phase $(\alpha \beta)$ system. Detailed information on the construction of the fictitious phase can be found in [15] and [16]. Although this technique achieves a desired harmonic compensation, its implementation has some drawbacks that include the following: (1) the use of the Hilbert transform to construct the fictitious phase requires high computational load, (2) given the sensitivity of the algorithm to grid frequency fluctuations, any variation in the grid frequency introduces an error in the calculated reference current, and (3) reactive power compensation is not considered in this method. To overcome some of the aforementioned drawbacks, Kim et al. proposed a control technique that is referred in this paper as the double synchronous reference frame (DSRF) method [17]. In the DSRF method, the fictitious phase is constructed by using a low-pass filter and two SRFs $\left(\mathrm{dq}^{+}\right.$and $\left.\mathrm{dq}^{-}\right)$rotating in opposite directions to generate the reference current. The succeeding section demonstrates that, from a control point of view, the DSRF method is equivalent to the control technique depicted in Fig. 2, except that the fictitious phase ( $\beta$-axis signal) is set to zero. Poor performance in reactive power compensation, unnecessary complexity, and high computational burden for the control system are main drawbacks of the DSRF method.

As previously discussed, the fictitious current signal is a common feature in all previously reported RCG techniques in SRF. This paper therefore proposes a novel RCG technique in SRF, which does not require a fictitious current signal and exhibits frequency-independent operation, accurate reference current extraction, and relatively fast transient response.

A detailed analysis of the proposed strategy under different operating conditions of a shunt APF is presented in this study. The results indicate the superior performance of the proposed method. The theoretical evaluations are confirmed through experiments.

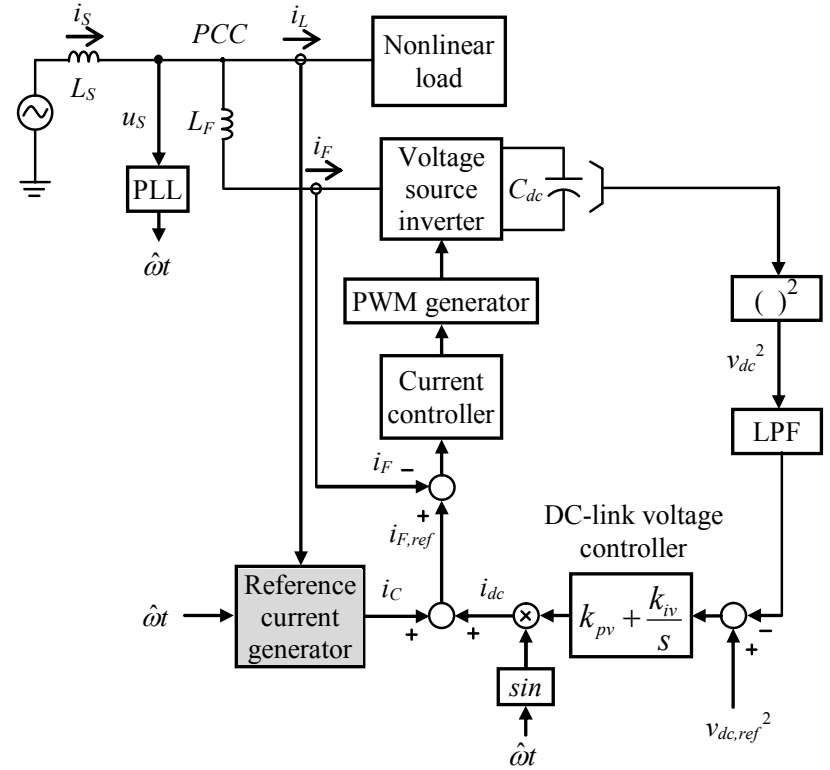

Fig. 1. Schematic diagram of a single-phase shunt APF.

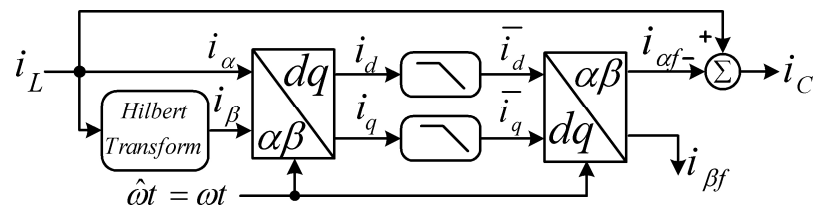

Fig. 2. Basic scheme of the SRF harmonic extraction technique [14].

\section{REVIEW OF DSRF METHOD AND INTRODUCTION TO PROPOSED METHOD}

Fig. 3 shows the basic scheme of the DSRF method [17]. $i_{\alpha f}$ and $i_{C}$ are the extracted fundamental and harmonic components of the non-linear load current, respectively.

Moving the sums ahead of LPFs simplifies the DSRF method, as shown in Fig. 4, from which the following equations are obtained:

$$
\begin{aligned}
i_{d} & =i_{d, p o s}-i_{d, \text { neg }} \\
i_{q} & =i_{q, p o s}+i_{q, \text { neg }}
\end{aligned}
$$

The $\alpha \beta$ reference frame is transformed into $\mathrm{dq}^{+}$and $\mathrm{dq}^{-}$ reference frames, as expressed in the following matrix equations [17]:

$$
\begin{aligned}
& {\left[\begin{array}{l}
i_{d, p o s} \\
i_{q, p o s}
\end{array}\right]=T(t)\left[\begin{array}{l}
i_{\alpha} \\
i_{\beta}
\end{array}\right]} \\
& {\left[\begin{array}{l}
i_{d, n e g} \\
i_{q, \text { neg }}
\end{array}\right]=T(-t)\left[\begin{array}{c}
i_{\alpha} \\
i_{\beta}
\end{array}\right]}
\end{aligned}
$$

where

$$
T(t)=\left[\begin{array}{cc}
\sin (\omega t) & -\cos (\omega t) \\
-\cos (\omega t) & -\sin (\omega t)
\end{array}\right]
$$

By substituting (3) and (4) into (1) and (2), $i_{d}$ and $i_{q}$ are written as follows: 


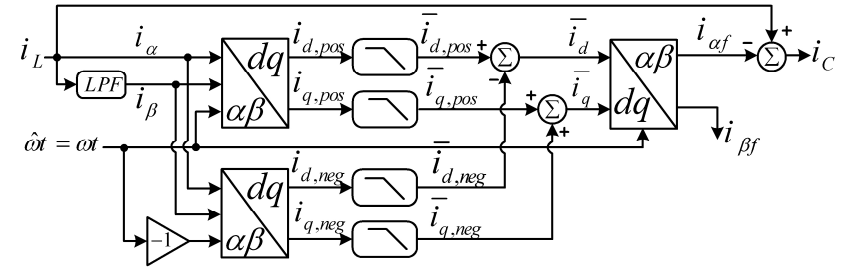

Fig. 3. Basic scheme of the DSRF method [17].

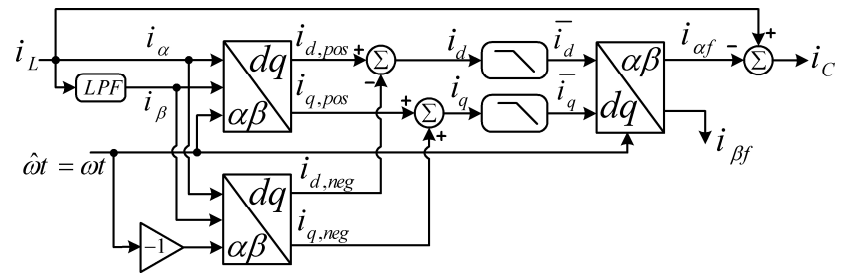

Fig. 4. Simplification of the DSRF method using block diagram algebra.

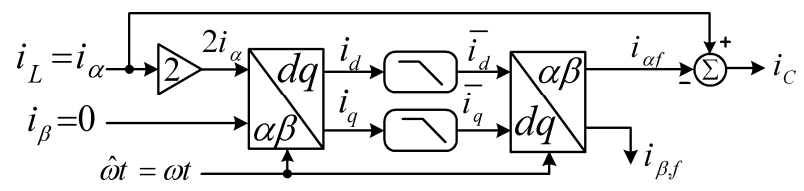

Fig. 5. Simplified equivalent of the DSRF method.

$$
\begin{aligned}
& i_{d}=2 \sin (\omega t) i_{\alpha} \\
& i_{q}=-2 \cos (\omega t) i_{\alpha}
\end{aligned}
$$

In matrix form, (6) and (7) are rewritten as

$$
\left[\begin{array}{l}
i_{d} \\
i_{q}
\end{array}\right]=\left[\begin{array}{cc}
\sin (\omega t) & -\cos (\omega t) \\
-\cos (\omega t) & -\sin (\omega t)
\end{array}\right]\left[\begin{array}{c}
2 i_{\alpha} \\
0
\end{array}\right]=T(t)\left[\begin{array}{c}
2 i_{\alpha} \\
0
\end{array}\right]
$$

Using the above matrix equation, the DSRF method illustrated in Fig. 4 can be further simplified to that which is exhibited in Fig. 5.

From the control point of view, the control structure shown in Fig. 5 is the exact equivalent of the DSRF technique presented in Fig. 3. Therefore, the DSRF method imposes unnecessary complexity and high computational burden to the control system.

The drawbacks of the DSRF method are further investigated by performing a simple mathematical analysis described in the succeeding paragraphs.

The non-linear load current is represented by [11]

$$
i_{L}=I_{m} \sin (\omega t-\phi)+\sum_{k=3,5,7, . .} I_{k} \sin \left(k \omega t-\phi_{k}\right)
$$

where $\omega$ is the grid voltage fundamental frequency, $I_{m}$ and $\phi$ are the amplitude and the phase of the fundamental current, respectively, and $I_{k}$ and $\phi_{k}$ are the magnitude and the phase of the $k^{\text {th }}$ harmonic-current component, respectively.

By substituting (9) into (6) and (7), $i_{d}$ and $i_{q}$ are obtained as

$$
\begin{aligned}
& i_{d}=I_{m} \cos (\phi)-I_{m} \cos (2 \omega t-\phi) \\
& +\sum_{k=3,5,7, . .} I_{k}\left[\cos \left((k-1) \omega t-\phi_{k}\right)-\cos \left((k+1) \omega t-\phi_{k}\right)\right] \\
& i_{q}=I_{m} \sin (\phi)-I_{m} \sin (2 \omega t-\phi) \\
& -\sum_{k=3,5,7, \ldots} I_{k}\left[\sin \left((k-1) \omega t-\phi_{k}\right)-\sin \left((k+1) \omega t-\phi_{k}\right)\right]
\end{aligned}
$$

where the constant terms $I_{m} \cos (\phi)$ and $I_{m} \sin (\phi)$ correspond to the magnitude of the active and of the reactive currents, respectively, of the non-linear load.

From (10) and (11), $i_{d}$ and $i_{q}$ obviously contain significant second-order harmonic content. Thus, the removal of these variables requires the use of a first- or a second-order LPF with low cut-off frequency or of a high-order LPF. A high-order LPF imposes high computational load to the control system, in addition to stability problems [18]. By contrast, using a low-order LPF with low cut-off frequency significantly degrades the transient performance of APF.

With the foregoing analysis, the efficiency of the control technique reported by Kim et al. [17] is significantly undermined.

To cancel out undesired double-frequency oscillations without degrading the stability and the transient performance of the system, a novel method called the double-frequency oscillation cancellation (DFOC) method is presented in this paper. It is worth mentioning that the cancellation of double-frequency oscillations is already attended in unbalanced three-phase systems [19]. The performance of this approach is investigated by conducting a detailed mathematical analysis.

\section{DFOC METHOD}

This section mainly focuses on eliminating double-frequency ripples $I_{m} \cos (2 \omega t-\phi) \quad$ and $I_{m} \sin (2 \omega t-\phi)$ from $i_{d}$ and $i_{q}$, respectively. Thus, for the sake of simplicity, $i_{d}$ and $i_{q}$ are considered as

$$
\begin{aligned}
& i_{d}=I_{m} \cos (\phi)-I_{m} \cos (2 \omega t-\phi) \\
& i_{q}=I_{m} \sin (\phi)-I_{m} \sin (2 \omega t-\phi)
\end{aligned}
$$

Expanding (12) and (13) gives

$$
\begin{aligned}
& i_{d}=I_{m} \cos (\phi)-I_{m} \cos (\phi) \cos (2 \omega t)-I_{m} \sin (\phi) \sin (2 \omega t) \\
& i_{q}=I_{m} \sin (\phi)+I_{m} \sin (\phi) \cos (2 \omega t)-I_{m} \cos (\phi) \sin (2 \omega t)
\end{aligned}
$$

The amplitudes of the double-frequency oscillation terms obviously depend on the average values of $i_{d}$ and $i_{q}$. The perfect cancellation of these undesired components can be easily achieved by injecting double-frequency signals with the same amplitude, except the opposite phase angle, into $i_{d}$ and $i_{q}$. Fig. 6 illustrates the schematic diagram of the proposed strategy, where $\hat{\omega}$ denotes the estimated frequency by PLL. 
In this section, we assume that $\hat{\omega}=\omega$. The LPF block is subsequently considered as a first-order LPF, expressed as follows:

$$
\operatorname{LPF}(s)=\frac{\omega_{c}}{s+\omega_{c}}
$$

where $\omega_{c}$ is the cut-off frequency of LPF.

To analyze the performance of the proposed method, the mathematical expressions for $\overline{i_{d}}$ and for $\overline{i_{q}}$ are derived.

$$
\begin{aligned}
& \overline{i_{d}}=A_{d}+\left\{B_{d} \cos (\omega t) \cos \left(\omega_{f} t\right)\right. \\
& +C_{d} \sin (\omega t) \sin \left(\omega_{f} t\right) \\
& +D_{d} \sin (\omega t) \cos \left(\omega_{f} t\right) \\
& \left.+E_{d} \cos (\omega t) \sin \left(\omega_{f} t\right)\right\} e^{-\omega_{C} t} \\
& \overline{i_{q}}=A_{q}+\left\{B_{q} \cos (\omega t) \cos \left(\omega_{f} t\right)\right. \\
& +C_{q} \sin (\omega t) \sin \left(\omega_{f} t\right) \\
& +D_{q} \sin (\omega t) \cos \left(\omega_{f} t\right) \\
& \left.+E_{q} \cos (\omega t) \sin \left(\omega_{f} t\right)\right\} e^{-\omega_{\mathcal{C}} t}
\end{aligned}
$$

where

$$
\begin{aligned}
& \omega_{f}=\sqrt{\omega^{2}-\omega_{c}^{2}}, \\
& A_{d}=-B_{d}=-D_{q}=I_{m} \cos (\phi), \\
& C_{d}=-E_{q}=-I_{m}\left[\omega \cos (\phi)+\omega_{c} \sin (\phi)\right] / \omega_{f}, \\
& D_{d}=A_{q}=-B_{q}=I_{m} \sin (\phi), \text { and } \\
& E_{d}=C_{q}=-I_{m}\left[\omega_{c} \cos (\phi)+\omega \sin (\phi)\right] / \omega_{f} .
\end{aligned}
$$

Appendix A presents the detailed derivation of (17) and (18). To highlight the advantages of the proposed method, the mathematical expressions for $\overline{i_{d}}$ and for $\overline{i_{q}}$ without using the DFOC block are also presented. These expressions are as follows:

$$
\begin{aligned}
& \overline{i_{d}}=I_{m} \cos (\phi)-\left(A_{d}^{\prime}+I_{m} \cos \phi\right) e^{-\omega_{C} t} \\
& +A_{d}^{\prime} \cos (2 \omega t)+B_{d}^{\prime} \sin (2 \omega t) \\
& \overline{i_{q}}=I_{m} \sin (\phi)-\left(A_{q}^{\prime}+I_{m} \sin \phi\right) e^{-\omega_{c} t} \\
& +A_{q}^{\prime} \cos (2 \omega t)+B_{q}^{\prime} \sin (2 \omega t)
\end{aligned}
$$

where

$$
\begin{aligned}
& A_{d}^{\prime}=B_{q}^{\prime}=I_{m} \omega_{c}\left(2 \omega \sin \varphi-\omega_{c} \cos \phi\right) /\left(4 \omega^{2}+\omega_{c}^{2}\right), \text { and } \\
& B_{d}^{\prime}=-A_{q}^{\prime}=-I_{m} \omega_{c}\left(2 \omega \cos \phi+\omega_{c} \sin \varphi\right) /\left(4 \omega^{2}+\omega_{c}^{2}\right) .
\end{aligned}
$$

From (17) and (18), the fluctuating terms obviously decay to zero with a time constant of $1 / \omega_{c}$ and $\overline{i_{d}}$ and $\overline{i_{q}}$ converge to $I_{m} \cos (\phi)$ and $I_{m} \sin (\phi)$, respectively. However, in (19) and (20), $\bar{i}_{d}$ and $\overline{i_{q}}$ contain high-amplitude, double-frequency contents. These results are clearly illustrated in Fig. 7 for $I_{m}=10 \mathrm{~A}, \phi=\pi / 3 \mathrm{rad}, \omega_{c}=50 \mathrm{rad} / \mathrm{s}$, and $\omega=100 \pi \mathrm{rad} / \mathrm{s}$. As expected, perfect cancellation of double-frequency is achieved when the DFOC block is used.

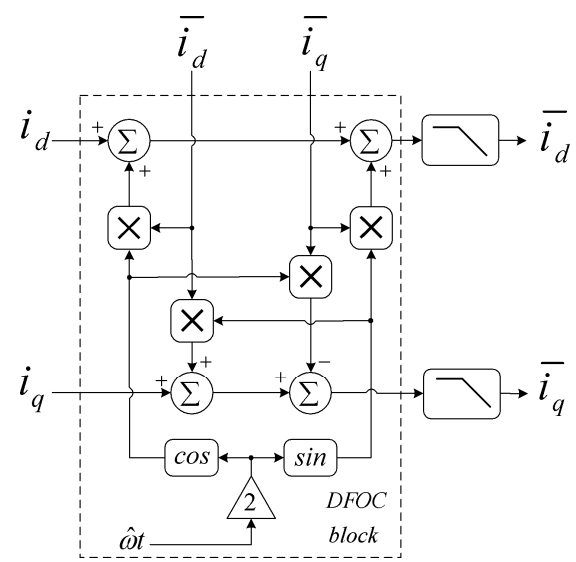

Fig. 6. Schematic diagram of the DFOC method.

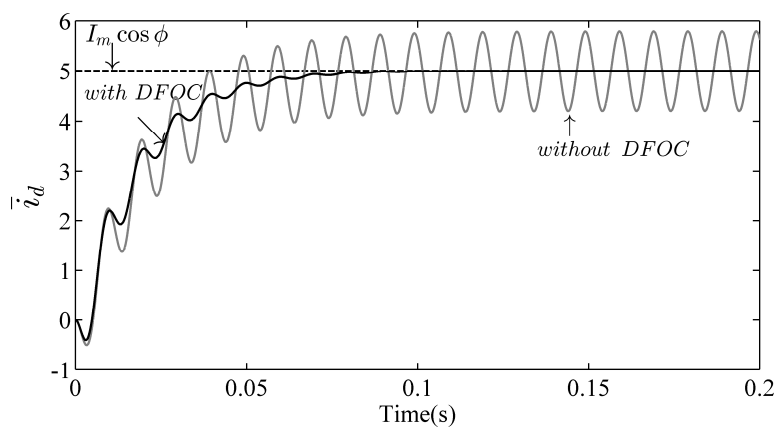

(a)

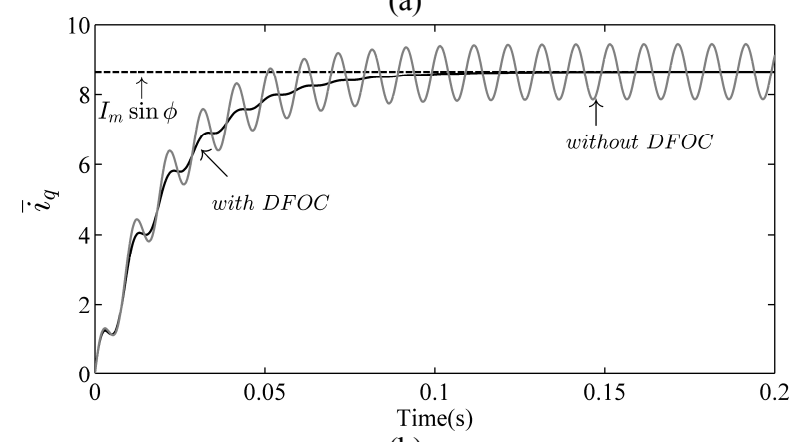

(b)

Fig. 7. Comparative results for $\overline{i_{d}}$ and $\overline{i_{q}}$. (a) $\overline{i_{d}}$ with (black line) and without (gray line) the use of the DFOC block. (b) $\bar{i}_{q}$ with (black line) and without (gray line) the use of the DFOC block.

\section{APPLICATION OF THE DFOC METHOD IN DiFFERENT CONTROL MODES OF A SHUNT APF}

This section investigates the application of the DFOC block in extracting the reference-compensating current in different control strategies of a shunt APF (i.e., UPF and PHC modes of operation).

\section{A. PHC Mode}

Fig. 8 shows the application of the DFOC block in extracting the reference-compensating current in the PHC mode of operation. The figure illustrates that the suggested strategy does not require a fictitious current signal. 


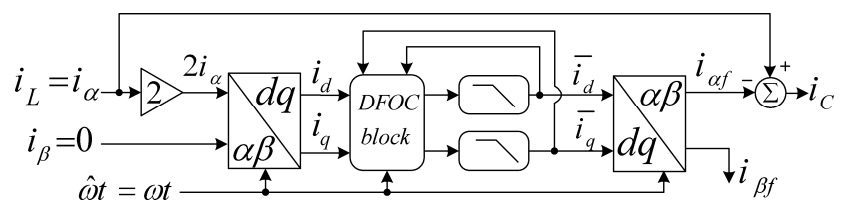

Fig. 8. Proposed method to extract the reference-compensating current in the PHC mode of a shunt APF.

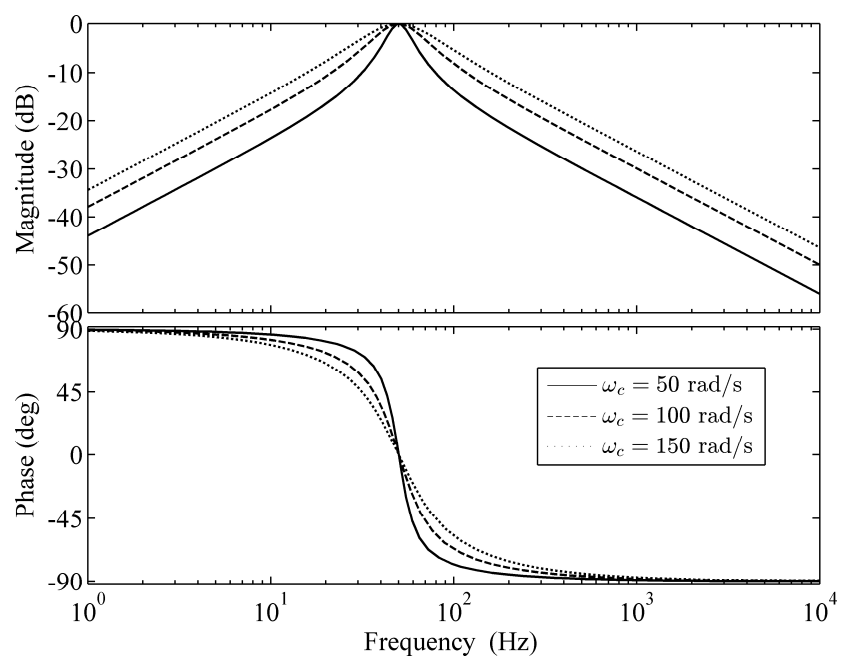

Fig. 9. Bode diagram of transfer function (21) for three different values of cut-off frequency $\omega_{c}$.

To evaluate the performance of the proposed method, the transfer function of the extracted fundamental current to the load current is expressed as follows:

$$
G_{D F O C}=\frac{i_{\alpha f}}{i_{L}}=\frac{2 \omega_{c} s}{s^{2}+2 \omega_{c} s+\omega^{2}}
$$

A detailed derivation of (21) can be found in Appendix B.

Fig. 9 shows the bode plot of transfer function (21) for three different values of cut-off frequency $\omega_{c}$. We observe that transfer function (21) exhibits a band-pass filtering behavior with a pass-band centered at the fundamental frequency. The bandwidth of the band-pass filter (BPF) is proportional to the cut-off frequency of LPF. The lower the cut-off frequency, the less is the BPF bandwidth and the better is the filtering performance.

The transient behavior of the proposed algorithm can be analyzed by identifying the closed loop poles of transfer function (21), which are written as follows:

$$
p_{1}=-\omega_{c}+j \omega, p_{2}=-\omega_{c}-j \omega
$$

As shown in (22), the transient time is dictated by the cut-off frequency of LPF. The higher the cut-off frequency, the faster is the transient response, as stated in [20] and [21].

The dc-offset rejection capability is another important feature that must be examined. As expressed in (21), transfer function $G_{D F O C}$ has a zero located at the origin. Thus, the perfect rejection of the dc-offset is achieved for all values of $\omega_{c}$.

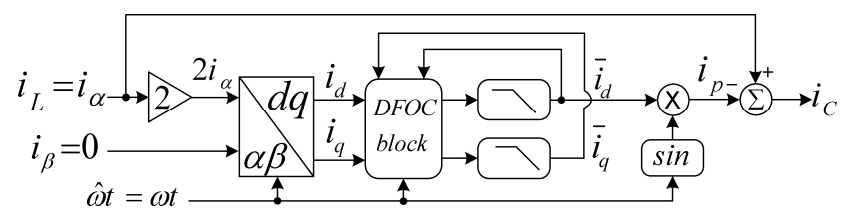

Fig. 10. Proposed method to extract the reference-compensating current in the UPF mode of a shunt APF.

Finally, the performance of the proposed algorithm is investigated under grid frequency deviations. Notice that the transfer function (21) is derived from the assumption that $\hat{\omega}=\omega$. Without this assumption, the transfer function of the extracted fundamental current to the load current can easily be demonstrated as

$$
G_{D F O C}=\frac{2 \omega_{c} s}{s^{2}+2 \omega_{c} s+\hat{\omega}^{2}}
$$

Substituting the Laplace operator $s=j \omega$ into (23) yields

$$
\left.G_{D F O C}\right|_{s=j \omega}=\frac{j 2 \omega_{c} \omega}{\hat{\omega}^{2}-\omega^{2}+j 2 \omega_{c} \omega}
$$

From (24), under variations in the grid frequency, if the estimated frequency by PLL is very close (or preferably equal) to the actual frequency, the fundamental-current component is perfectly extracted, ultimately achieving an accurate reference-compensating current.

\section{B. UPF Mode}

Fig. 10 shows the application of the DFOC block in extracting the reference-compensating current in the UPF mode of operation of a single-phase shunt APF, where $i_{p}$ denotes the extracted active current drawn by the load. By performing a procedure similar with that discussed in section IV-A, we can obtain the same operational characteristics for the system presented in Fig. 10, with the following: 1) frequency-independent operation and 2) dependence of the transient response and of the filtering accuracy to the cut-off frequency of LPF.

\section{OUTPUT CURRENT AND DC-LINK VOLTAGE CONTROLLERS}

The control scheme presented in Fig. 1 clearly indicates that the performance of the APF system highly depends on the quality of the current controller. If the converter model in Fig. 1 is simplified to $1 /\left(L_{F} S+R_{F}\right)$, where $L_{F}$ and $R_{F}$ are filter inductance and resistance, respectively, and assuming that the current controller is composed of $k_{p c}+k_{i c} / s$ (Fig. 11(a)), then the closed-loop transfer function of the current controller is easily derived as

$$
\frac{i_{F}}{i_{F, r e f}}=\frac{k_{p c} s+k_{i c}}{L s^{2}+\left(R+k_{p c}\right) s+k_{i c}}
$$

where $i_{F}$ and $i_{F, r e f}$ are the measured and the reference currents for APF, respectively. 
The choice of controller gains is obviously a compromise between the stability margin and the dynamic performance. In this study, the selected bandwidth is $2 \mathrm{kHz}$ that effectively covers the $39^{\text {th }}$ harmonics and is significantly lower than the switching frequency, thus preventing sensitivity to switching noises.

On the other hand, as depicted in Fig. 1, a controller must regulate the DC-link voltage to maintain the energy balance between APF and the grid. In practice, converter losses and leakage of DC-link capacitors cause the active filter to draw a fundamental active component that maintains the DC-link voltage at its pre-defined value [22]-[25]. The DC-link voltage controller is adopted from [3] in which the small-signal model of the DC-link voltage loop is derived as presented in Fig. 11(b), where $C_{d c}$ is the total DC-link capacitance, $U_{p s}$ is the peak value of the PCC voltage, $\omega_{c v}=60 \mathrm{rad} / \mathrm{s}$ is the cut-off frequency of the low-pass filter, and $k_{p v}$ and $k_{i v}$ are the gains of the PI regulator.

A significantly lower bandwidth for the voltage loop than that of the current loop is selected to avoid interaction between the DC-link voltage and the output current controllers. Indeed, a bandwidth that is not larger than one tenth of the grid frequency and a phase margin that ranges from $30^{\circ}$ to $60^{\circ}$ are recommended [3].

\section{EXPERIMENTAL RESULTS}

To confirm the performance of the proposed algorithm, the system shown in Fig. 1 is developed in the laboratory. A TMS320F28335 digital signal controller (DSC) from the Texas Instruments is used to perform the algorithm and to generate the proper gating signals for the single-phase converter. Table I shows the salient parameters of the system. For all experiments, the selected cut-off frequency of LPF is $95 \mathrm{rad} / \mathrm{s}$.

As a worst case scenario, the performance of the proposed APF system is investigated in the presence of a highly non-linear load consisting of a diode rectifier bridge feeding a capacitor in parallel with a resistor. As shown in Fig. 12, the load current is highly distorted with zero periods, while the source current remains sinusoidal and in-phase with the grid voltage. The current quality can be further improved by using the DFOC block. In this case, the total harmonic distortion (THD) is reduced to $4.18 \%$ and the lowest order harmonic $\left(3^{\text {rd }}\right.$ harmonic) is drastically attenuated.

In another study, as depicted in Fig. 13, a resistive load is being operated and the system is at a steady state. Suddenly, a non-linear rectifier load is switched on. The transient period is approximately two cycles of the fundamental frequency, which is relatively longer than that required for the FFT method by [26], almost equal to that in [27], and shorter compared with that in [28]. The source current and the grid voltage are initially in-phase and after the transient period, the phase difference is again converged to zero in the steady state, which means the unity power factor is achieved.

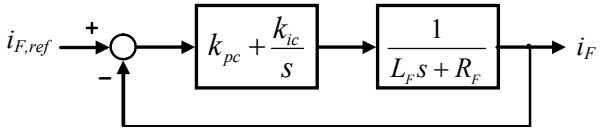

(a)

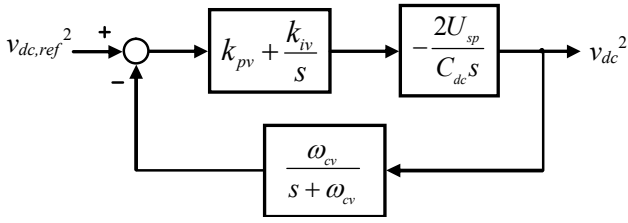

(b)

Fig. 11. Small-signal model of (a) output current and of (b) DC-link voltage loops [3].

TABLE I

SYSTEM PARAMETERS

\begin{tabular}{ccc}
\hline \hline Quantity & Description & Value \\
\hline$u_{s}$ & AC power source voltage (rms) & $110 \mathrm{~V}$ \\
$f$ & AC power source frequency & $50 \mathrm{~Hz}$ \\
$L_{s}$ & Grid inductance & $80 \mu \mathrm{H}$ \\
$C_{d c}$ & DC capacitance & $4800 \mu \mathrm{F}$ \\
$f_{s}$ & Switching frequency & $20 \mathrm{kHz}$ \\
$f_{s a m}$ & Sampling frequency & $40 \mathrm{kHz}$ \\
$L_{F}$ & Filter inductance & $2.5 \mathrm{mH}$ \\
$R_{F}$ & Filter resistance & $0.5 \Omega$ \\
\hline
\end{tabular}

TABLE II

THD AND TRANSIENT TIME OF THE SOURCE CURRENT FOR DIFFERENT CUT-OFF FREQUENCY VALUES OF LPF

\begin{tabular}{ccc}
\hline \hline$\omega_{c}(\mathrm{rad} / \mathrm{s})$ & THD (\%) & $\begin{array}{c}\text { Transient time } \\
(\mathbf{m s})\end{array}$ \\
\hline 50 & 1.91 & 45 \\
75 & 2.44 & 30 \\
100 & 2.50 & 24 \\
125 & 2.52 & 15 \\
150 & 2.75 & 12 \\
\hline
\end{tabular}

- Simple algorithm

- A fictitious current signal is not required

- Frequency-independent operation

- Accurate reference-current extraction

- Fast transient response

Table II summarizes the transient time and THD of the source current with the same resistive plus rectifier load used for Fig. 13 and for different cut-off frequency values of LPF. As expected, the higher the cut-off frequency, the faster is the transient response and THD is higher.

In the last study, shown in Fig. 14, the load on the system is abruptly changed to approximately $200 \%$ and then the DC-link voltage recovers in around $400 \mathrm{~ms}$, exhibiting a smooth transient performance and confirming the slow bandwidth with sufficient stability margin of the DC-link voltage loop. 


\section{CONCLUSIONS}

A novel method in the synchronous reference frame for extraction of the reference-compensating current for single-phase APFs is proposed in this study. The major advantages of this technique are summarized as follows:

The effectiveness of the proposed method is investigated by conducting a detailed mathematical analysis and is confirmed by the experiments.

\section{APPENDIX}

A. Derivation of Mathematical Expressions for $\bar{i}_{d q}$ [Fig. 6]

From Fig. 6, in the Laplace domain, we obtain

$$
\begin{aligned}
& {\overline{i_{d}}}_{d}(s)=\frac{\omega_{c}}{s+\omega_{c}} \times \ell\left[i_{d}(t)+\overline{i_{d}}(t) \cos (2 \omega t)+\bar{i}_{q}(t) \sin (2 \omega t)\right](26) \\
& {\overline{i_{q}}}_{q}(s)=\frac{\omega_{c}}{s+\omega_{c}} \times \ell\left[\bar{i}_{q}(t)+\overline{i_{d}}(t) \sin (2 \omega t)-\bar{i}_{q}(t) \cos (2 \omega t)\right](27)
\end{aligned}
$$

where $\ell$ denotes the Laplace operator.

Multiplying both sides of (26) and of (27) by $\left(s+\omega_{c}\right)$ and rearranging the results yield

$$
\begin{aligned}
& \overline{i_{d}}(s)=\omega_{c} \times \ell\left[i_{d}(t)+(\cos (2 \omega t)-1) \overline{i_{d}}(t)+\sin (2 \omega t) \bar{i}_{q}(t)\right](28) \\
& \overline{i_{q}}(s)=\omega_{c} \times \ell\left[i_{q}(t)+\sin (2 \omega t) \overline{\bar{i}_{d}}(t)-(\cos (2 \omega t)+1) \overline{\bar{i}_{q}}(t)\right](29)
\end{aligned}
$$

Converting (28) and (29) into the time-domain and rearranging the results into matrix form yield

$$
\begin{array}{r}
{\left[\begin{array}{c}
\dot{\overline{i_{d}}}(t) \\
\dot{\overline{i_{q}}}(t)
\end{array}\right]=\omega_{c}\left[\begin{array}{cc}
\cos (2 \omega t)-1 & \sin (2 \omega t) \\
\sin (2 \omega t) & -\cos (2 \omega t)-1
\end{array}\right]\left[\begin{array}{l}
\overline{i_{d}}(t) \\
\overline{i_{q}}(t)
\end{array}\right]} \\
+\left[\begin{array}{cc}
\omega_{c} & 0 \\
0 & \omega_{c}
\end{array}\right]\left[\begin{array}{l}
i_{d}(t) \\
i_{q}(t)
\end{array}\right]
\end{array}
$$

Substituting (12) and (13) into (30) and rearranging the results yield

$$
\begin{array}{r}
{\left[\begin{array}{c}
\dot{\overline{i_{d}}}(t) \\
\dot{\overline{i_{q}}}(t)
\end{array}\right]=\omega_{c}\left[\begin{array}{cc}
\cos (2 \omega t)-1 & \sin (2 \omega t) \\
\sin (2 \omega t) & -\cos (2 \omega t)-1
\end{array}\right]\left\{\left[\begin{array}{c}
\overline{\bar{i}_{d}}(t) \\
\bar{i}_{q}(t)
\end{array}\right]\right.} \\
\left.-\left[\begin{array}{c}
I_{m} \cos (\phi) \\
I_{m} \sin (\phi)
\end{array}\right]\right\}
\end{array}
$$

From (31), the state-space description of the DFOC method can be derived as follows:

$$
\left\{\begin{array}{c}
\dot{x}(t)=A(t) x(t)+B(t) u(t) \\
y(t)=C \cdot x(t)
\end{array}\right.
$$

Where

$$
\begin{aligned}
& x(t)=\left[\begin{array}{c}
\bar{i}_{d}(t) \\
\overline{i_{q}}(t)
\end{array}\right] ; \quad u(t)=\left[\begin{array}{l}
I_{m} \cos (\phi) \\
I_{m} \sin (\phi)
\end{array}\right] \\
& A(t)=-B(t)=\omega_{c}\left[\begin{array}{cc}
\cos (2 \omega t)-1 & \sin (2 \omega t) \\
\sin (2 \omega t) & -\cos (2 \omega t)-1
\end{array}\right] \\
& C(t)=I
\end{aligned}
$$

The state-space equation (32) describes a two-input, two-output, and linear time-variant system. The analytical solution of (32) is highly complicated and is beyond the scope of this study. Thus, the final solution of the state-space equation (32), which is expressed in (17) and (18), is presented. Detailed information on solving the linear time-variant systems can be found in [20] and [21].

B. Determination of the Transfer Function between $i_{\alpha f}$ and $i_{L}$ [Fig. 8]

Substituting (6) and (7) into (30) yields

$$
\left[\begin{array}{c}
\dot{\overline{i_{d}}}(t) \\
\overline{\overline{i_{q}}}(t)
\end{array}\right]=A(t) \cdot\left[\begin{array}{c}
\overline{i_{d}}(t) \\
\overline{i_{q}}(t)
\end{array}\right]+2 \omega_{c}\left[\begin{array}{c}
\sin (\omega t) \\
-\cos (\omega t)
\end{array}\right]
$$

From Fig. 8, we obtain

$$
\left[\begin{array}{l}
\bar{i}_{d}(t) \\
\overline{i_{q}}(t)
\end{array}\right]=T(t)\left[\begin{array}{l}
i_{\alpha f}(t) \\
i_{\beta f}(t)
\end{array}\right]
$$

Substituting (33) into (32) gives

$$
\begin{aligned}
\frac{d}{d t}\left\{T(t) \cdot\left[\begin{array}{c}
i_{\alpha f}(t) \\
i_{\beta f}(t)
\end{array}\right]\right\}=A(t) T(t) \cdot & {\left[\begin{array}{l}
i_{\alpha f}(t) \\
i_{\beta f}(t)
\end{array}\right] } \\
& +2 \omega_{c}\left[\begin{array}{c}
\sin (\omega t) \\
-\cos (\omega t)
\end{array}\right] i_{L}
\end{aligned}
$$

Expanding the left-hand side term of (35), rearranging the results, and then multiplying both sides by $T(t)^{-1}$ yield

$$
\begin{array}{r}
\frac{d}{d t}\left[\begin{array}{l}
i_{\alpha f}(t) \\
i_{\beta f}(t)
\end{array}\right]=T(t)^{-1} \cdot\left(A(t) T(t)-\frac{d T(t)}{d t}\right) \cdot\left[\begin{array}{c}
i_{\alpha f}(t) \\
i_{\beta f}(t)
\end{array}\right] \\
+2 \omega_{c} T(t)^{-1}\left[\begin{array}{c}
\sin (\omega t) \\
-\cos (\omega t)
\end{array}\right] \cdot i_{L}
\end{array}
$$

where $T(t)^{-1}$ denotes the inverse of matrix $T(t)$.

After a number of simple mathematical manipulations, we derive

$$
\frac{d}{d t}\left[\begin{array}{l}
i_{\alpha f}(t) \\
i_{\beta f}(t)
\end{array}\right]=\left[\begin{array}{cc}
-2 \omega_{c} & -\omega \\
\omega & 0
\end{array}\right] \cdot\left[\begin{array}{c}
i_{\alpha f}(t) \\
i_{\beta f}(t)
\end{array}\right]+\left[\begin{array}{c}
2 \omega_{c} \\
0
\end{array}\right] \cdot i_{L}
$$

With $i_{\alpha f}$ as the output signal, the state-space representation of the proposed method can be expressed as

$$
\left\{\begin{array}{c}
\dot{x}(t)=A^{\prime}(t) x(t)+B^{\prime}(t) u(t) \\
y(t)=C^{\prime} x(t)
\end{array}\right.
$$

where

$$
\begin{aligned}
& x(t)=\left[\begin{array}{l}
i_{\alpha f}(t) \\
i_{\beta f}(t)
\end{array}\right] ; \quad u(t)=i_{L} \\
& A^{\prime}(t)=\left[\begin{array}{cc}
-2 \omega_{c} & -\omega \\
\omega & 0
\end{array}\right] ; B^{\prime}(t)=\left[\begin{array}{c}
2 \omega_{c} \\
0
\end{array}\right] \\
& C^{\prime}(t)=\left[\begin{array}{cc}
1 & 0
\end{array}\right]
\end{aligned}
$$

The state-space equation (38) describes a single-input, single-output, and linear time-invariant system. Thus, the system transfer function can be obtained as follows:

$$
G_{D F O C}=\frac{i_{\alpha f}(s)}{i_{L}(s)}=C^{\prime}\left[s I-A^{\prime}\right]^{-1} B^{\prime}=\frac{2 \omega_{c} s}{s^{2}+2 \omega_{c} s+\omega^{2}}
$$



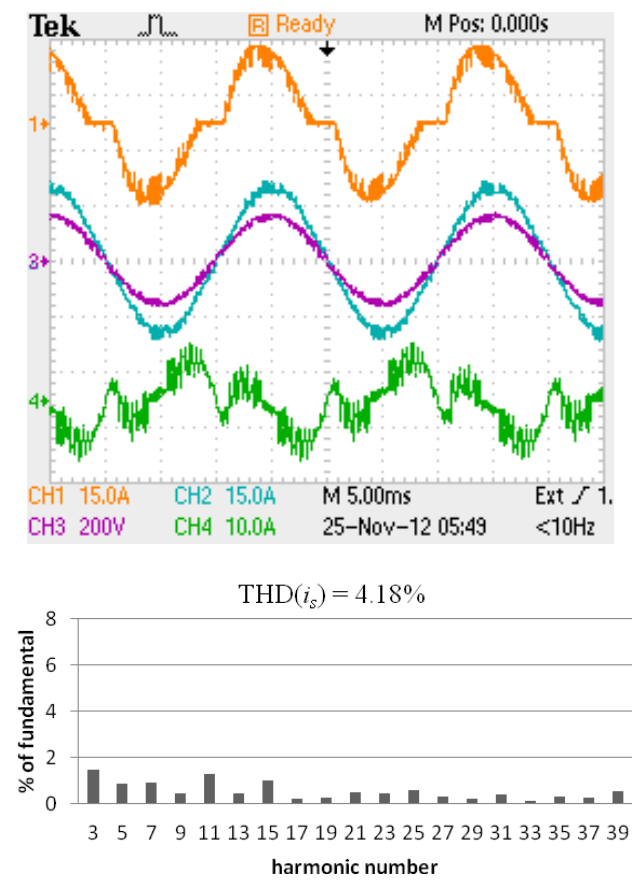

(a)
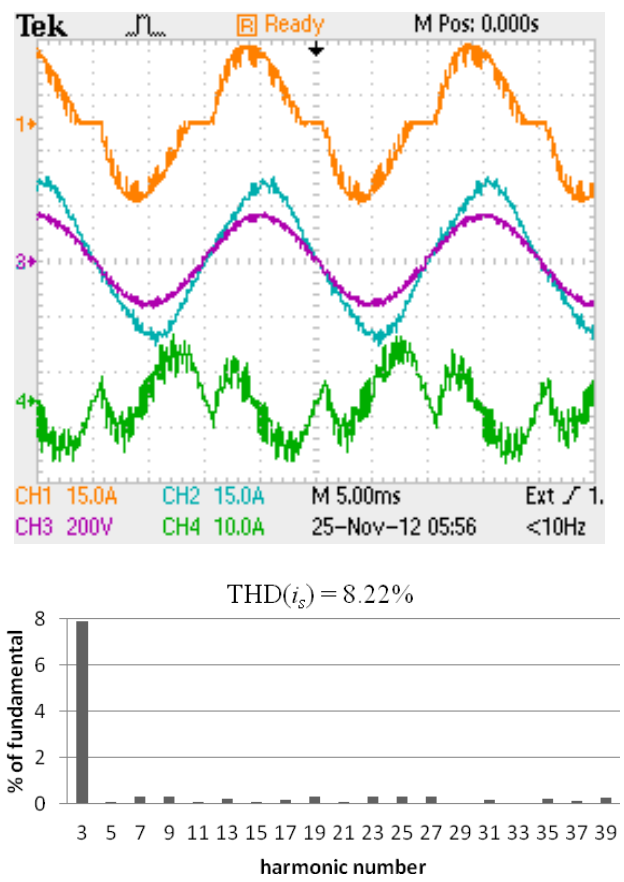

(b)

Fig. 12. Experimental waveforms and harmonic spectrum of the source current under a highly non-linear load (a) with and (b) without the use of the DFOC block: (CH1) load current, $(\mathrm{CH} 2)$ source current, $(\mathrm{CH} 3)$ grid voltage, and (CH4) APF output current.

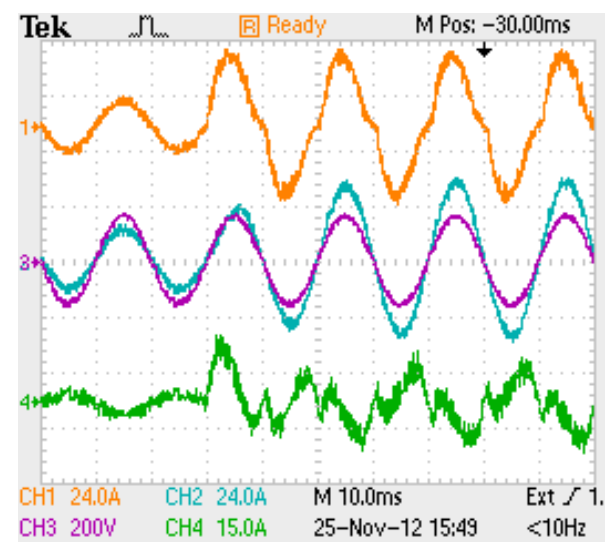

Fig. 13. Experimental waveforms when a rectifier load is added to the resistive load: (CH1) to $(\mathrm{CH} 4)$ as described in Fig. 12.

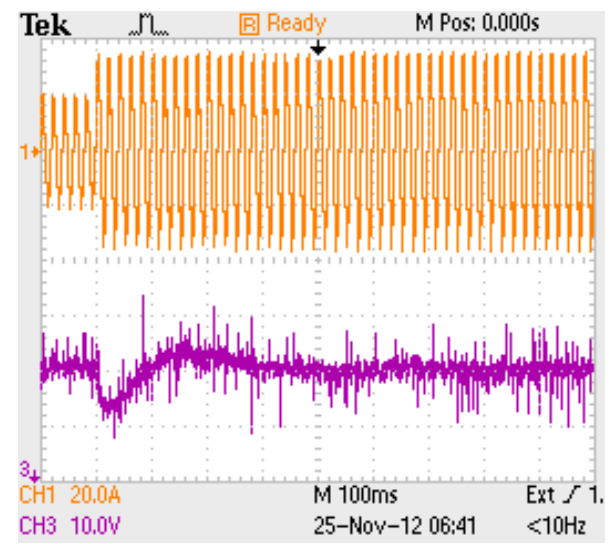

Fig. 14. Experimental waveforms in response to a step load change: $(\mathrm{CH} 1)$ load current and $(\mathrm{CH} 3)$ DC-link voltage.

\section{ACKNOWLEDGMENT}

This work was supported in part by the Research Deputy of Ferdowsi University of Mashhad under Grant no. 2/22211 (dated July 10, 2012). This support is gratefully acknowledged.

\section{REFERENCES}

[1] H. Akagi, "The state-of-the-art of active filters for power conditioning," in Proc. EPE '05, pp. P1-P15, 2005.

[2] B. Singh, Al-Haddad K., and A. Chandra, "A review of active power filters for power quality improvement," IEEE Trans. Ind. Electron., Vol. 45, No. 5, pp. 960-971, Oct. 1999.

[3] J. Miret, M. Castilla, J. Matas, J. M. Guerrero, and J. C. Vasquez, "Selective harmonic-compensation control for single-phase active power filter with high harmonic rejection," IEEE Trans. Ind. Electron., Vol. 56, No. 8, pp. 3117-3127, Aug. 2009.

[4] L. Ruomei, "A high power active filter for harmonic compensation in an electric locomotive," in Proc. PowerCon '02, pp. 1059-1063, 2002.

[5] V. Khadkikar, A. Chandra, and B. N. Singh, "Generalised single-phase $p-q$ theory for active power filtering: Simulation and DSP-based experimental investigation," IET Power Electron., Vol. 2, No. 1, pp. 67-78, Jan. 2009.

[6] M. Cirrincione, M. Pucci, and G. Vitale, "A single-phase DG generation unit with shunt active power filter capability by adaptive neural filtering," IEEE Trans. Ind. Electron., Vol. 55, No. 5, pp. 2093-2110, May 2008.

[7] K. R. Uyyuru, M.K. Mishra, and A. Ghosh, "An optimization-based algorithm for shunt active filter under distorted supply voltages," IEEE Trans. Power Electron., 
Vol. 24, No. 5, pp. 1223-1232, May 2009.

[8] M. Rukonuzzaman and M. Nakaoka, "Single-phase shunt active power filter with harmonic detection," in Proc. Electr. Power Appl. '02, pp. 343-350, 2002.

[9] B. M. Han, B. Y. Bae, and S. J. Ovaska, "Reference signal generator for active power filters using improved adaptive predictive filter," IEEE Trans. Ind. Electron., Vol. 52, No. 2, pp. 576-584, Apr. 2005.

[10] P. Kumar and A. Mahajan, "Soft computing techniques for the control of an active power filter," IEEE Trans. Power Del., Vol. 24, No. 1, pp. 452-461, Jan. 2009.

[11] F. D. Freijedo, J. D. Gandoy, O. Lopez, P. F. Comesana, and C. M. Penalver, "A signal-processing adaptive algorithm for selective current harmonic cancellation in active power filters," IEEE Trans. Ind. Electron., Vol. 56, No. 8, pp. 2829-2840, Aug. 2009.

[12] L. P. Kunjumuhammed and M. K. Mishra, "Comparison of single phase shunt active power filter algorithms," in Proc. Annu. Conf. IEEE Power India, 2006.

[13] S. A. Gonzalez, R. G. Retegui, and M. Benedetti, "Harmonic computation technique suitable for active power filters," IEEE Trans. Ind. Electron., Vol. 54, No. 5, pp. 2791-2796, Oct. 2007.

[14] M. Saitou, N. Matsui, and T. Shimizu, "A control strategy of single phase active filter using a novel $\mathrm{d}-\mathrm{q}$ transformation," in Proc. IEEE IAS '03, pp. 1222-1227, 2003.

[15] J.-W. Choi, Y.-K. Kim, and H.-G. Kim. "Digital PLL control for single-phase photovoltaic system," in Proc. Electr. Power Appl. '06, pp. 40-46, 2006.

[16] S. M. Silva, B. M. Lopes, B. J. C. Filho, R. P. Campana, and W. C. Bosventura, "Performance evaluation of PLL algorithms for single phase grid-connected systems," in Proc. IAS '04, pp. 2259-2263, 2004.

[17] J. S. Kim, and Y. S. Kim, "A new control method for a single-phase hybrid active power filter based on a rotating reference frame," Journal of Power Electronics, Vol. 9, No. 5, pp. 718-725, Sep. 2009.

[18] C. H. Ng, K. Busawon, G. A. Putrus, and L. Ran, "Fast-individual-harmonic-extraction technique," in Proc. Gener. Transm. Distrib. '05, pp. 556-562, 2005.

[19] P. Rodriguez, J. Pou, J. Bergas, J.I. Candela, R. P. Burgos, and D. Boroyevich, "Decoupled double synchronous reference frame PLL for power converters control," IEEE Trans. Power Electron., Vol. 22, No. 2, pp. 584-592, Mar. 2007.

[20] T. Kailath, Linear Systems, Prentice-Hall, New York, 1980.

[21] C. T. Chen, Introduction to Linear System Theory, Holt Rinehart and Winston Inc., New York, 1970.

[22] Y. Han, and L. Xu, "Design and implementation of a robust predictive control scheme for active power filters," Journal of Power Electronics, Vol. 11, No. 5, pp. 751-758, Sep. 2011.

[23] J. H. Lee, J. K. Jeong, B. M. Hany, and B. Y. Bae, "New reference generation for a single-phase active power filter to improve steady state performance," Journal of Power Electronics, Vol. 10, No. 4, pp. 412-418, Jul. 2010

[24] H. H. Tumbelaka, L. J. Borle, C. V. Nayar, and S. R. Lee, "A grid current-controlling shunt active power filter," Journal of Power Electronics, Vol. 9, No. 3, pp. 365-376, May 2009.

[25] M. Adel, S. Zaid, and O. Mahgoub, "Improved active power filter performance based on an indirect current control technique," Journal of Power Electronics, Vol. 11,
No. 6, pp. 931-937, Nov. 2011.

[26] L. Asiminoaei, F. Blaabjerg, and S. Hansen, "Detection is key-Harmonic detection methods for active power filter applications," IEEE Ind. Appl. Mag., Vol. 13, No. 4, pp. 22-33, Jul./Aug. 2007.

[27] M. K. Ghartemani, H. Mokhtari, M. R. Iravani, and M. Sedighy, "A signal processing system for extraction of harmonics and reactive current of single phase systems", IEEE Trans. Power Deliv., Vol. 19, pp. 979-986, Jul. 2004.

[28] J. S. Tepper, J. W. Dixon, G. Venegas, and L. Morán, "A simple frequency-independent method for calculating the reactive and harmonic current in a non-linear load," IEEE Trans. Power Electron., Vol. 43, pp. 647-653, Dec. 1996.

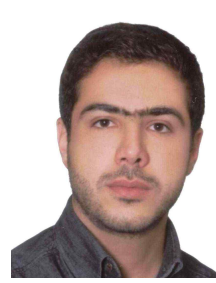

Mohammad Monfared received his B.Sc. degree in Electrical Engineering from the Ferdowsi University of Mashhad in Iran in 2004, and his M.Sc. and Ph.D. degrees in Electrical Engineering (both with honors) from the Amirkabir University of Technology in Tehran, Iran in 2006 and in 2010, respectively. He is currently an Assistant Professor at the Ferdowsi University of Mashhad, Iran. His research interests include power electronics, motor drives, renewable energy systems, energy conversion, and control and applications.

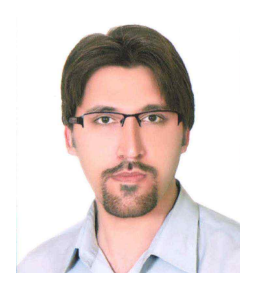

Saeed Golestan received his B.Sc. degree in Electrical Engineering from the Shahid Chamran University in Ahvaz, Iran in 2006, and his M.Sc. degree in Electrical Engineering from the Amirkabir University of Technology in Tehran, Iran in 2009. He is currently a Lecturer in the Department of Electrical Engineering at the Islamic Azad University (Abadan branch) in Iran. His research interests include power quality and distributed generation systems.

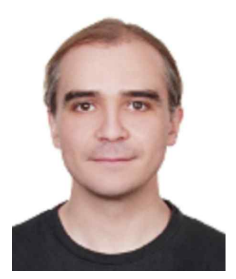

Josep M. Guerrero was born in Barcelona, Spain in 1973. He received his B.S. degree in Telecommunications Engineering, M.S. degree in Electronics Engineering, and Ph.D. degree in Power Electronics from the Technical University of Catalonia in Barcelona, Spain in 1997, 2000, and 2003, respectively. He was an Associate Professor in the Department of Automatic Control Systems and Computer Engineering at the Technical University of Catalonia where he currently teaches courses on digital signal processing, field-programmable gate arrays, microprocessors, and renewable energy. Since 2004, he has been responsible for the Renewable Energy Laboratory at the Escola Industrial de Barcelona. He has been a visiting professor at Zhejiang University in Hangzhou, China and at the University of Cergy-Pontoise in Pontoise, France. In 2012, he was a guest professor and chair at the Nanjing University Aeronautics and Astronautics. Since 2011, he has been a full professor of microgrids in the Institute of Energy Technology at the Aalborg University in Aalborg, Denmark, where he is the responsible of the microgrids research program. His research interests include different aspects of microgrids, such as power electronics, distributed energy storage systems, hierarchical and cooperative control and energy management systems, and optimization of microgrids and islanded minigrids. 